\begin{tabular}{|c|l|}
\hline Title & A Iveolar echinococcosis of the liver in children \\
\hline Author(s) & $\begin{array}{l}\text { Yoshida, Tadashi; Kamiyama, Toshiya; Okada, Tadao; Nakanishi, Kazuaki; Y okoo, Hideki; Kamachi, Hirofumi; } \\
\text { Matsushita, Michiaki; Sato, Naoki; Sasaki, Fumiaki; Todo, Satoru }\end{array}$ \\
\hline Citation & $\begin{array}{l}\text { Journal of Hepato-Biliary-Pancreatic Sciences, 17(2), 152-157 } \\
\text { https://doi.org/L0.1007/300534_009-011466 }\end{array}$ \\
\hline Issue Date & 2010-03 \\
\hline Doc URL & http://hdl.handle.net/2115/43105 \\
\hline Rights & The original publication is available at www.springerlink.com \\
\hline Type & article (author version) \\
\hline File Information & JHBPS17-2_152-157.pdf \\
\hline
\end{tabular}

Instructions for use 


\section{Alveolar Echinococcosis of the Liver in Children}

${ }^{1}$ Department of Digestive and General Surgery, Hokkaido University Graduate School of Medicine, Kita-ku, North 15, west 7, Sapporo $060-8638$, Japan

${ }^{2}$ Department of Pediatric Surgery, Hokkaido University Graduate School of Medicine, Sapporo, Japan

Tadashi Yoshida ${ }^{1}$, Toshiya Kamiyama ${ }^{1}$, Tadao Okada², Kazuaki Nakanishi ${ }^{1}$, Hideki Yokoo $^{1}$, Hirofumi Kamachi ${ }^{1}$, Michiaki Matsushita ${ }^{1}$, Naoki Sato ${ }^{1}$, Fumiaki Sasaki ${ }^{2}$, and Satoru Todo ${ }^{1}$

Address reprint requests to: Tadashi Yoshida, MD; Department of Digestive and General Surgery, Hokkaido University Graduate School of Medicine, Kita-ku, Kita 15, Nishi 7, Sapporo 060-8638, Japan

TEL : +81-11-706-5927 FAX: +81-11-717-7515

E-mail: ytadashi324@yahoo.co.jp

Short title: Alveolar Echinococcosis.

Running title: Alveolar Echinococcosis of the Liver in Children.

Keywords: child, alveolar echinococcosis, liver 


\section{Abstract}

Background/Purpose. Alveolar echinococcosis of the liver $(A E L)$ is a zoonosis that is distributed in cold regions of the northern hemisphere. The disease is mostly found in adults and rarely in pediatric patients because it tends to be slow growing.

Patients and Methods. Ten Japanese pediatric patients with AEL (under fifteen-year-old) have been operated on in Hokkaido University Hospital from January 1936 to June 2008. We examined these children and revealed the characteristics of AEL. Results. The patients included three males and seven females whose mean age was 10.9 years old, ranging from 7 to 15 . The length of follow-up was from 3 months to 33 years (median: 19 years). Six cases were picked up by mass screening and nine cases who underwent hepatectomy are still alive and one case whose tumors were unresectable died of liver failure.

Conclusion. Our cases indicate that some AEL pediatric patients advanced rapidly, so early detection is imperative. Thus, screening examinations are essential for children in contaminated areas, and complete radical resection should be performed if a liver tumor is found on a screening examination and diagnosed as AEL. 


\section{Introduction}

Echinococcosis of the liver is a zoonosis caused by tape worms belonging to the genus Echinococcus (family Taeniidae). Echinococcus is mainly classified into two groups, Echinococcus granulosus and Echinococcus multilocularis, which cause cystic echinococcosis and alveolar echinococcosis, respectively. While Echinococcus granulosus is distributed widely throughout the world, Echinococcus multilocularis is distributed in cold regions of the nor thern hemisphere such as Europe, Russia, North America, and Japan. ${ }^{1,2}$ Alveolar echinococcosis is found in the majority of echinococcosis cases in Japan, which is found almost only in Hokkaido in northern Japan. ${ }^{2,3}$ Because alveolor echinococcosis of the liver ( $A E L$ ) tends to be slow growing, the disease is mostly found in adults and rarely in pediatric patients. It has been reported that patients should receive early treatment because AEL often invades or metastasizes to other organs like a malignant neoplasm. ${ }^{4}$ As far as we know, only two case reports of AEL in children have been published in Japan, except for our cases, and there have been no other summary reports of $A E L$ in children. ${ }^{2,5}$ We report ten cases of $A E L$ in Japanese children in our hospital.

\section{Patients and Methods}




\section{Patients}

Ten pediatric Japanese patients with AEL (under fifteen years old) have been operated on in Hokkaido University Hospital from January 1936 to June 2008 .

2. Methods

We performed checkups and revealed the epidemiological characteristics, clinicopathological findings, treatments and clinical courses of pediatric patients with AEL. Mass screening for AEL have been performed in Hokkaido, Japan. The previous mass screening method utilized serological tests in the first step, which were complement fixation test (CF), indirect hemagglutination (IHA) and immunoelectrophoresis (IE) until 1983. Physical examination for hepatomegaly and examination for calcification by X-ray were performed in the second step. From 1984, enzyme-linked immunosorbent assay (ELISA) was introduced as the primary serological screening test and ultrasonography (US) has been adopted for those patients who give positive results by ELISA as the second step examination. ${ }^{6}$ AEL was diagnosed by serological tests such as ELISA and Western-blotting (WB) and imaging methods such as X-ray, US, computed tomography (CT) and magnetic resonance imaging (MRI).

\section{Results}

Ten patients were pathologically confirmed as AEL. These 
included three males and seven females whose mean age was 10.9 years old, ranging from 7 to 15 . This makes up $3.8 \%$ of all operated AEL patients (263 cases) in our hospital.

The habitation areas of the patients were spread widely throughout Hokkaido, Japan. Six patients lived in Nemuro and Kushiro sub-prefectures (eastern Hokkaido), two in Oshima (southern Hokkaido), and two in Ishikari and Kamikawa (central Hokkaido) .

Eight AEL patients were asymptomatic and their method of diagnosis were mass screening (six cases) and close examinations for enuresis (two cases). Only two patients were symptomatic with symptoms such as fever, abdominal pain, and general fatigue. No patients had any past or family histories of AEL.

While three patients considered drinking spring water or well water as the transmission route, the other seven patients had not drank spring water and it was unknown how they had been infected.

At the time of diagnosis, the staging system proposed by Nakajima et al was used. ${ }^{7}$ There were 2, 4, 0,2 , and 2 cases in stages $I, \mathbb{I}, \mathbb{I I} a, \mathbb{I} b$, and $\mathrm{IV}$, respectively (Table 1 ). We utilized serological tests and imaging methods as a means of diagnosing AEL. CF, IHA, and IE were performed for 6, 6, and 5 patients, including 6,2 , and 2 positive, respectively. ELISA was performed for six patients, including two positive and four 
suspected positive cases. WB was performed for four patients and all of them were positive. All of the patients had abdominal X-ray examinations and abnormal calcifications of the right upper abdomen were detected in six patients. Abdominal US and CT were performed for seven patients and cystic lesions and/or calcifications of the liver were detected in the seven patients, respectively. The US and CT findings of AEL are categorized into four patterns, respectively, by Sasaki et al. ${ }^{8}$ One patient underwent MRI and small round cysts with a weakly enhanced solid component were detected (Table 2) .9

The operative procedures were hepatectomy in eight patients, including two cases with hepatectomy with resection of other organs, exploratory laparotomy in one, and marsupialization in one (Table 3).

The length of follow-up was from 3 months to 33 years (median: 19 years). All of the ten patients, except for case 1 , are still alive (Table 4). Case 1 had diffuse unresectable foci of AEL at the time of diagnosis and exploratory laparotomy was done. In spite of chemotherapy by thymol ester of palmitic acid after surgery, she died of liver failure combined with progression of AEL three years later. Case 3 underwent marsupialization for drainage because of a large tumor that occupied almost the entire left lobe of the liver, and extended left lobectomy was performed as a two-staged operation. Case 4 underwent partial hepatectomy (S5, S6, S8). She needed partial hepatectomy again 
because of stump recurrence after a four-year interval from the first operation. Case 7 underwent extended left lobectomy and para-aorta lymph node dissection. She was not treated with chemotherapy after surgery despite residual para-aorta lymph node metastasis because the safety of albendazole for pediatric patients was not confirmed at the time. Case 9 underwent partial hepatectomy and resection of the diaphragm and abdominal wall. She was treated with albendazole after surgery, taking care with strict examinations, because of the infiltration of the abdominal wall and uncertainty of dissemination. She is alive and free from relapse. Case 10 was ten-year-old boy and examinations for enuresis were performed. Abdominal CT showed the calcifications on the s8 of the liver (two lesions). ELISA, WB test, US, and MRI were performed and he was diagnosed as AEL (Figure 1). He underwent partial hepatectomy (S8) (Figure 2, $3)$.

\section{Discussion}

AEL is often called lethal parasitosis because it can lead to death if left untreated and the progression of the disease is classified as follows: incubative, advanced, and end period. The length of the incubative period is considered to be five to fifteen years. As patients were likely to be asymptomatic 
during the incubative period, the disease was often discovered in more advanced periods in the past. The progression of AEL was considered to be slow, but AEL in pediatric patients can advance rapidly according to our cases, especially as seen in case 1 .

Although, CF, IHA, and IE had been used for mass screening, they were not appropriate for mass screening because they could often give false-negative for some patients in early stage..$^{3,6}$ Since the primary medical examination by ELISA and the secondary by US were introduced in 1984 in Hokkaido, Japan, AEL is now sometimes found in early stages. ${ }^{10}$ The absorbance of antibody to multilocular echinococcus antigen (ELISA value) was detected on ELISA and the criteria of ELISA values are as follows: positive $\geq 1.00$, negative $\leq 0.50$ and suspected positive $0.51<$ $\odot .99 .{ }^{10,11}$ The antibodies to multilocular echinococcus antigen such as $C$ antigen are detected in WB. ${ }^{10}$ The calcifications and cysts of the liver on US and CT have been widely used as characteristic findings and now MRI is considered to be useful for diagnosis. ${ }^{8,9}$ It is reported that the MRI findings of AEL are small round cysts with a weakly enhanced solid component and the cystic component can be a large and/or irregular lesion, and such lesions are depicted clearly on T2-weighted MRI by Kodama et al. ${ }^{9}$ Thus, it is hoped that MRI plays an important role in non invasive examinations. In Hokkaido, the primary medical examination by ELISA is done for applicants older than 
the third-year student and candidates who are classified into positive or suspected positive should undergo a secondary medical examination. In our case, a seven-year-old girl died of liver failure because the foci of AEL occupied the entire liver and was unresectable at diagnosis. This indicates that screening examination is essential even for children in contaminated areas and complete examination for AEL should be done if a liver tumor is found on a screening examination and diagnosed as AEL.

The only treatment for pediatric and adult AEL cases is radical resection, and nine cases whose primary lesions were completely resected were alive at the most recent follow up. In surgery, the ideal surgical margin is at least $1 \mathrm{~cm}$ in our hospital because the foci of AEL do not have any capsules and the borders of the foci are discontinuous and irregular. ${ }^{12}$ Case 4 had a stump recurrence after four years from the first operation and re-resection was performed. She is still alive and free from relapse twenty-four years after the second surgery. This indicates that complete resection is very important. Despite the fact that para-aortic lymph node metastasis was unresectable during the primary operation in case 7 , she has not progressed over the twenty-eight years after the surgery. It is noteworthy that radical resection of the primary lesion can offer long-term survival even if metastatic foci are residual. 
Albendazole, one of the benzimidazole carbamates, is often administrated to adult patients with invasion to other organs, dissemination, and non curative resection or palliative operation. It was found that albendazole reduced the foci of $A E L$ in six of fourteen cases with non curative resections and two of five palliative operations, respectively, by Ishizu et al. ${ }^{13}$ It is reported that adverse effects were mild and included one case each of mild elevation of serum transaminase level and alopecia in adult patients. However, there has not been extensive experience of albendazole in pediatric patients. ${ }^{13,14}$ In case 9, the AEL tumor ruptured and peritoneal dissemination was suspected before the operation. She was prescribed albendazole and has taken it for two and half years after surgery without any adverse effects or recurrences. It remains possible that albendazole could prevent relapse for pediatric patients in cases of non curative resection or palliative operation. Actually, we have not laid down the strict indication of the drug therapy for pediatric patients of AEL. Because there have been no reports of the effect of thymol ester of palmitic acid for AEL patients since 1965, the effect has been uncertain over the last few decades and we have not prescribed it after case $2 .{ }^{15}$ We prescribe albendazole for pediatric patients of AEL only in cases of non curative resection or palliative operation under the strict monitoring of adverse effects (general condition and laboratory data) after the report on albendazole by Ishizu et 
al in $1997 .{ }^{13}$

We report ten cases of $A E L$ in pediatric patients. Although it was thought that the foci of AEL tended to grow slowly, our cases indicate that some cases of AEL in pediatric patients can advance rapidly. In summary, a screening examination is essential for children in contaminated areas, and complete radical resection should be performed if a liver tumor is found on a screening examination and diagnosed as AEL. 


\section{References}

1) Rausch RL. On the ecology and distribution of Echinococcus spp. (Cestoda Taeniidae) and characteristics of their development in the intermediate host. Ann Parasitol Hum Comp $1967 ; 42: 19-63$.

2) Sugai M, Kimura N, Ishido K, Munakata H, Hakamada K, Hiroshi Satoh, et al. A case report of Alveolar Echinococcus with pulmonary lesions in a child (in Japanese with English abstract). Nihon Shoni-Geka Gakkai Zasshi (Jpn J Pediatr Surg) $2005 ; 41(2): 237-242$.

3) Sasaki F, Hata $Y$, Sato N, Sato $Y$, Igarashi M, Kameda H, et al. Alveolar hydatid disease of the liver in children (in Japanese with English abstract). Nihon Shoni-Geka Gakkai Zasshi (Jpn J Pediatr Surg) 1989; 25(1): 47-52.

4) Sasaki F, Hata Y, Sato N, Hamada H, Uchino J. Alveolar Echinococcosis of the liver in children. Hokkaido University Medical Library Series 1993; 30: 131-136.

5) Mizumoto M, Ishikawa N. A seven-year-old boy with Echinococcosis found by scattered calcification in the liver (in Japanese with English abstract). Nihon Shonikagaku Zasshi (Jpn J Pediatr) 1997; 101(6): 1071-1075.

6) Sato N, Uchino J, Suzuki K, Kamiyama T, Takahashi M, Shimamura T, et al. Mass screening. Hokkaido University Medical Library Series 1993; 30: 121-129. 
7) Nakajima Y, Sato N, Uchino J. Stage of the disease. Hokkaido University Medical Library Series 1993; 30: 115-119

8) Sasaki F, Ohkawa $Y$, Sato N, Uchino J, Hata $Y$. Imaging diagnosis of alveolar echinococcosis in young patients. Pediatr Radiol 1997; 27: 63-66.

9) Kodama Y, Fujita N, Shimizu T, Endo H, Nambu T, Sato N, et al. Alveolar echinococcosis: MR findings in the liver. Radiology 2003; 228: 172-177.

10) Furuya K, Sato N, Uchino J. Enzyme-linked immunosorbent assay (ELISA) and Western blotting (WB) test. Hokkaido University Medical Library Series 1993; 83: 75-91.

11) Sato H, Mitamura H, Arai J, Kumagai M. Serological diagnosis of human hydatid diseases by enzyme-linked immunosorbent assay (Part 1) Enzyme-linked immunosorbent assay by multilocular Echinococcus antigen (in Japanese with English abstract). Report of the Hokkaido Institute of Public Health 1983; 33: 8-15.

12) Uchino J, Sato N, Nakajima Y, Matsushita M, Takahashi M, Une Y. Treatment. Hokkaido University Medical Library Series $1993 ; 30: 137-149$.

13) Ishizu H, Uchino J, Sato N, Aoki S, Suzuki K, Kuribayashi H. Effect of albendazole on recurrent and residual alveolar echinococcosis of the liver after surgery. Hepatology 1997; 25(3): 528-531.

14) Sato N, Ishizu H, Aoki S, Akabane H, Watanabe I, Takahashi 
M, et al. Effectiveness of albendazole therapy for alveolar echinococcosis of the liver patients (in Japanese with English abstract). Shinryo to Shinyaku 1995; 32: 135-145. 15) Plotnikov NN, Ozeretskovskaia NN, Alekseeva MI, Turchins ME, Vitebskii laD, Diakin VM et al. Use of tepal (thymol ester of palmitic acid) in human echinococcosis (in Russian). Sov Med 1965; 28: 129-136. 


\section{Figure Legends}

Figure 1. MRI shows two lesions of AEL. (a)T1-weighted image obtained with small round cysts in the 58 of the liver (arrow). (b) Half-Fourier acquisition single-shot turbo spin echo (HASTE) image obtained with small high intensity lesions in the $\mathrm{s} 8$ of the liver (arrow).

Figure 2. The aggregated small calcifications were found on the surface of the liver (S8) during the operation (arrows).

Figure 3 . The resected specimen shows solid components with micro cysts. 


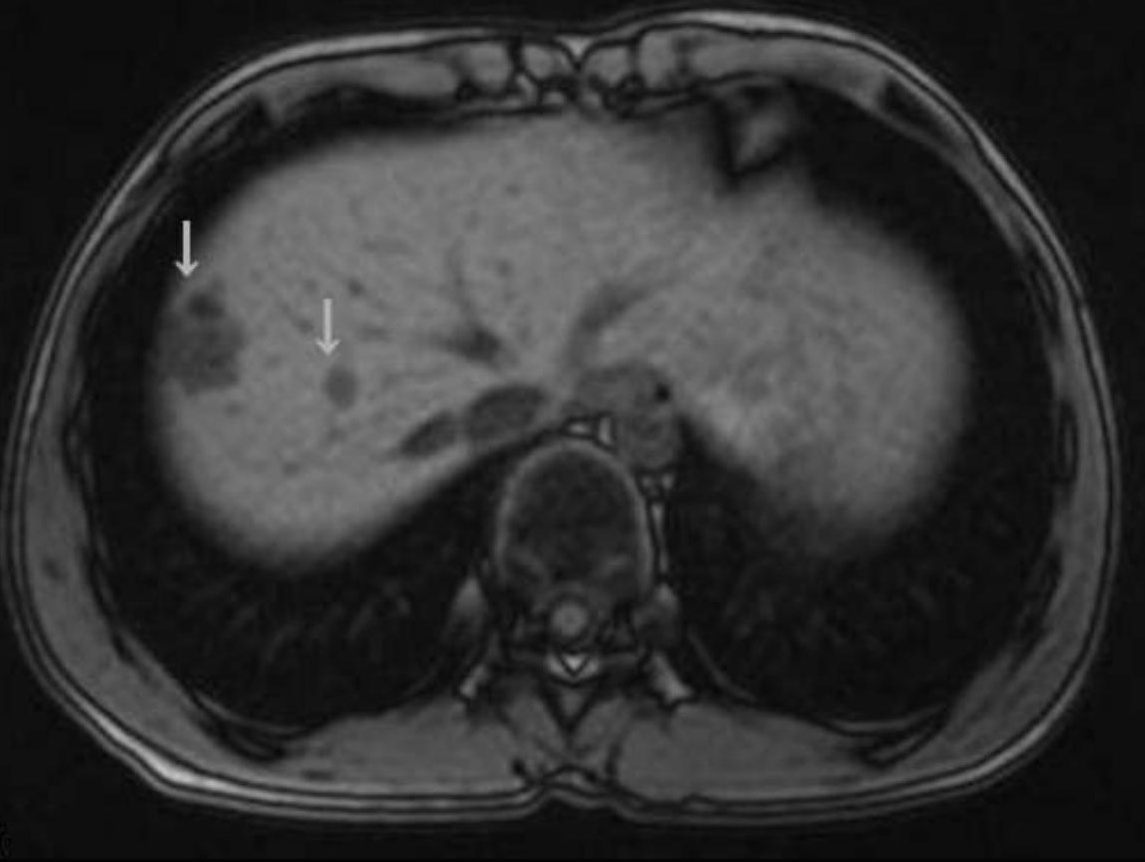





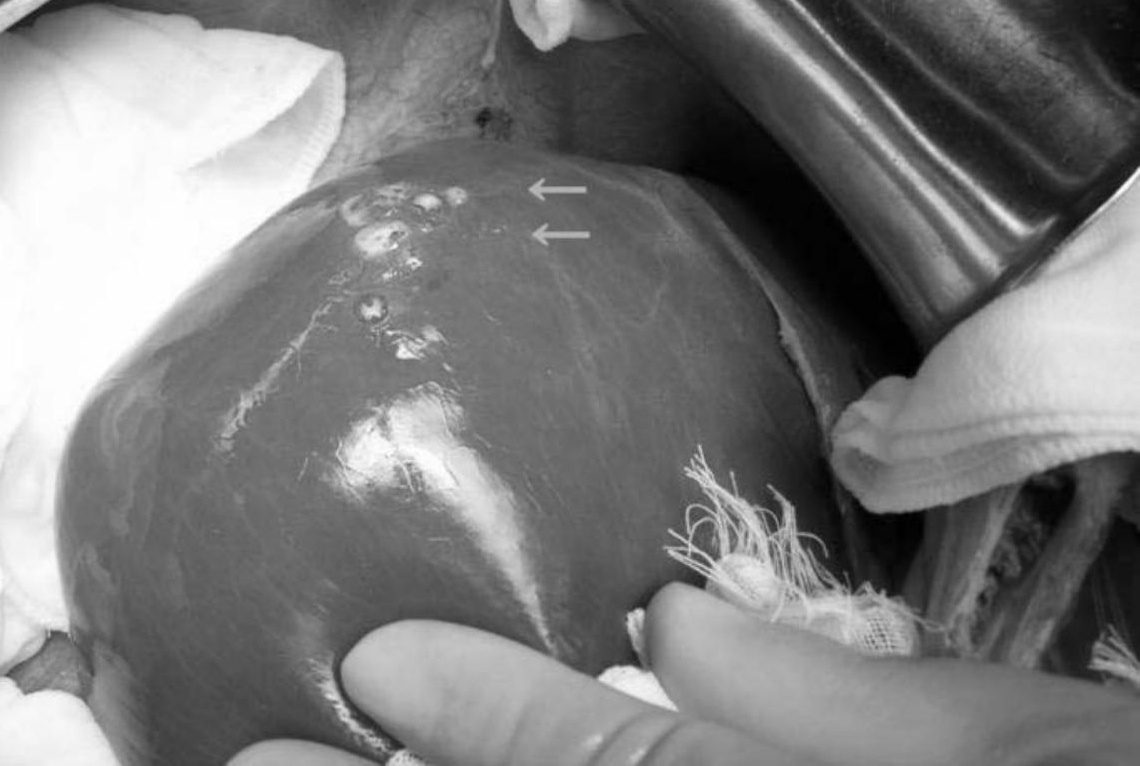




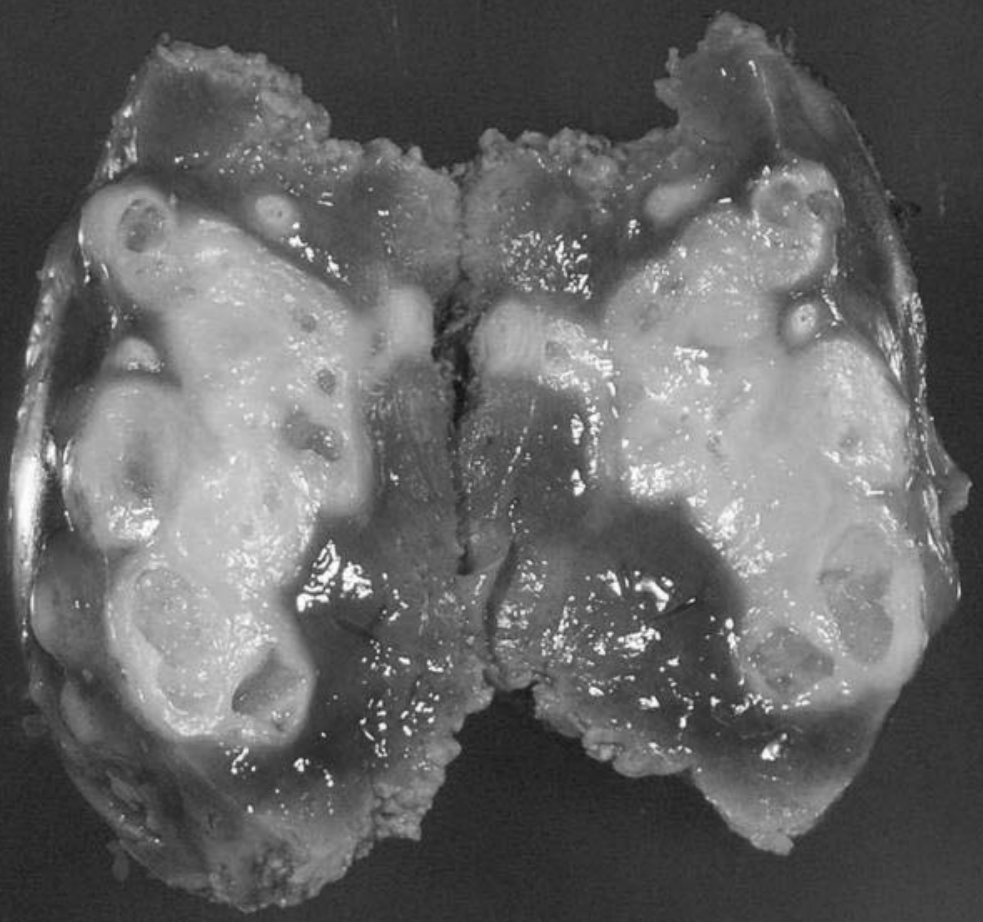

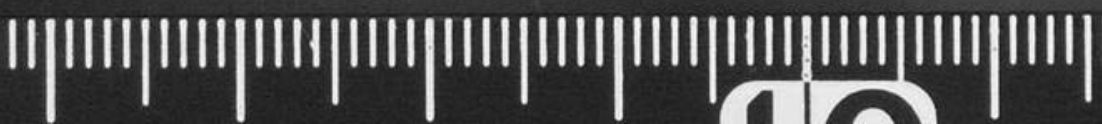


Table 1 Condition at the time of diagnosis

\begin{tabular}{|c|c|c|c|c|c|c|c|c|c|c|}
\hline Case & Year & Age & Sex & Habitation area & Chief complaint & Initial opportunity for diagnosis & Past history & Family history & Spring water & Stage* \\
\hline 1 & 1966 & 7 & $\mathrm{~F}$ & Nemuro & fever, abdominal pain & examination for hepatomegaly & morbilli, scarlatina & - & unknown & IV \\
\hline 2 & 1975 & 15 & $M$ & Nemuro & none & mass screening & - & Father: tuberculosis & + & I \\
\hline 3 & 1977 & 13 & M & Nemuro & none & mass screening & - & - & + & III $b$ \\
\hline 4 & 1980 & 15 & $\mathrm{~F}$ & Nemuro & none & mass screening & unknown & unknown & unknown & II \\
\hline 5 & 1987 & 8 & $\mathrm{~F}$ & Oshima & none & mass screening & unknown & unknown & unknown & II \\
\hline 6 & 1988 & 12 & $\mathrm{~F}$ & Kushiro & none & mass screening & unknown & unknown & unknown & II \\
\hline 7 & 1990 & 10 & $\mathrm{~F}$ & Oshima & none (enuresis) & examination for enuresis & - & - & - & IV \\
\hline 8 & 1997 & 10 & $\mathrm{~F}$ & Nemuro & none & mass screening & - & - & + & II \\
\hline 9 & 2005 & 9 & $\mathrm{~F}$ & Ishikari & fever, fatigue & examination for fever and fatigue & - & - & - & III $b$ \\
\hline 10 & 2008 & 10 & $M$ & Kamikawa & none (enuresis) & examination for enuresis & - & - & - & I \\
\hline
\end{tabular}

* Staging system proposed by Nakajima 
Table 2 Diagnostic method

\begin{tabular}{ccccccccccc}
\hline Case & CF & IHA & IE & ELISA & ELISA value & WB & X-ray & US & CT & MRI \\
\hline 1 & + & N.D. & N.D. & N.D. & - & N.D. & $*$ & N.D. & N.D. & N.D. \\
2 & + & + & + & N.D. & - & N.D. & $*$ & N.D. & N.D. & N.D. \\
3 & + & + & + & N.D. & - & N.D. & $*$ & N.D. & N.D. & N.D. \\
4 & + & - & - & N.D. & - & N.D. & $*$ & $\dagger 1$ & $\neq 1$ & N.D. \\
5 & + & - & - & \pm & 0.74 & N.D. & - & $\dagger 1$ & $\neq 1$ & N.D. \\
6 & + & - & - & + & unknown & N.D. & $*$ & $\dagger 1$ & $\neq 1$ & N.D. \\
7 & N.D. & N.D. & N.D. & + & 1 & + & $*$ & $\dagger 4$ & $\neq 1$ & N.D. \\
8 & N.D. & N.D. & N.D. & \pm & 0.82 & + & - & $\dagger 2$ & $\neq 2$ & N.D. \\
9 & N.D. & N.D. & N.D. & \pm & 0.896 & + & - & $\dagger 4$ & $\neq 4$ & N.D. \\
10 & N.D. & N.D. & N.D. & \pm & 0.643 & + & - & $\dagger 1$ & $\neq 1$ & $\S$
\end{tabular}

CF; complement fixation test, IHA; indirect hemagglutination, IE; immunoelectrophoresis ELISA; enzyme-linked immunosorbent assay, WB; Western-Blotting

US; ultrasonography, CT; computed tomography

MRI; magnetic resonance imaging, N.D. ; not done

* Calcification on abdominal X-ray

$\dagger 1$ Granular hyperechoic foci with or without acoustic shadow

†2 Irregular echogenic stroma, i.e., inflammatory or necotic changes are more echogenic than normal liver parenchyma and less echogenic than calcified parenchyma

†3 Small clustered vesicles called metacestodes detected as small hypoechoic areas with irregular margins

†4 Large anechoic or hypoechoic areas of liquefaction necrosis and/or cysts which are occasionally accompanied by an irregular internal echo

(†1 to †4: US finding categories proposed by Sasaki)

₹1 Focal high attenuation areas of calcification

₹2 Irregular low attenuation areas

¥3 Small, low attenuation cysts less than $2 \mathrm{~cm}$ in diameter

¥4 Large, low attenuation cysts more than $2 \mathrm{~cm}$ in diameter

( $\neq 1$ to $¥ 4$ : CT finding categories proposed by Sasaki)

$\S$ Small round cysts with a weakly enhanced solid component 
Table 3 Operation

\begin{tabular}{|c|c|c|c|}
\hline Case & Location* & Invasion / metastasis & Operative procedure \\
\hline 1 & APML & - & Simple laparotomy \\
\hline 2 & $\mathrm{~L}$ & - & Lateral segmentectomy \\
\hline 3 & AML & Stomach, diaphragm & Marsupialization + extended left lobectomy \\
\hline 4 & PA & - & Partial hepatectomy $\times 2$ \\
\hline 5 & PA & - & Right lobectomy \\
\hline 6 & PA & - & Right lobectomy \\
\hline 7 & PAM & Para-Aorta lymph node & Extended right lobectomy + lymph node dissection \\
\hline 8 & PA & - & Right lobectomy \\
\hline 9 & AP & Diaphragm, abdominal wall & Partial hepatectomy + Diaphragm and abdominal wall resection \\
\hline 10 & A & - & Partial hepatectomy \\
\hline
\end{tabular}

* A; anterior segment of the liver, $\mathrm{P}$; posterior, $\mathrm{M}$; middle, L; lateral 
Table 4 Post operative therapy and outcome

\begin{tabular}{|c|c|c|c|}
\hline Case & Residual lesion & Post operative therapy & Years since operation/outcome \\
\hline 1 & primary lesion & thymol ester of palmitic acid & 3/Died \\
\hline 2 & - & - & 33/Alive \\
\hline 3 & - & - & 31/Alive \\
\hline 4 & - & - & 28/Alive \\
\hline 5 & - & - & $21 /$ Alive \\
\hline 6 & - & - & 20/Alive \\
\hline 7 & Para-Aorta Lymph node & - & 18/Alive \\
\hline 8 & - & - & 11/Alive \\
\hline 9 & suspect of peritoneal dissemination & albendazole & 2/Alive \\
\hline 10 & - & - & 3months/Alive \\
\hline
\end{tabular}

